\title{
Imaging the Replication of Single Viruses: Lessons Learned from HIV and Future Challenges To Overcome
}

\author{
Susana Rocha,* Jelle Hendrix, Doortje Borrenberghs, Zeger Debyser,* and Johan Hofkens
}

Cite This: https://dx.doi.org/10.1021/acsnano.0c06369

ABSTRACT: The molecular composition of viral particles indicates that a single virion is capable of initiating an infection. However, the majority of viruses that come into contact with cells fails to infect them. Understanding what makes one viral particle more successful than others requires visualizing the infection process directly in living cells, one virion at a time. In this Perspective, we explain how single-virus imaging using fluorescence microscopy can provide answers to unsolved questions in virology. We discuss fluorescent labeling of virus particles, resolution at the subviral and molecular levels, tracking in living cells, and imaging of interactions between viral and host proteins. We end this Perspective with a set of remaining questions in understanding the life cycle of retroviruses and how imaging a single virus can help researchers address these questions. Although we use examples from the HIV field, these methods are of value for the study of other viruses as well.

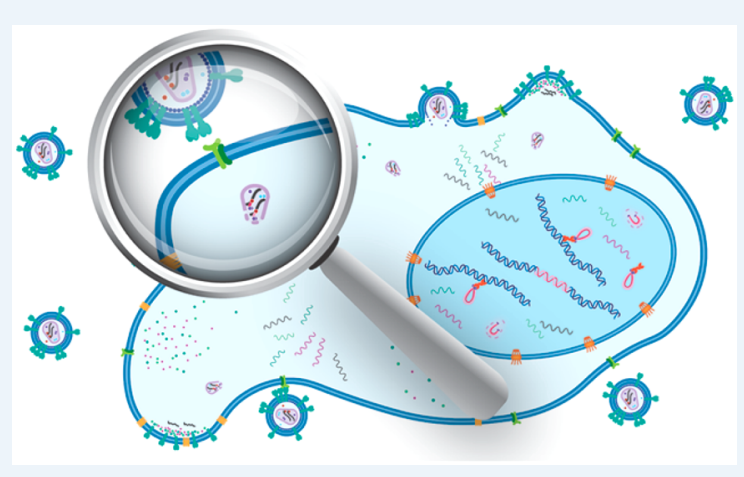

$\mathrm{V}$ iruses are small genetic entities that completely depend on infecting host cells to reproduce. Retroviruses are enveloped RNA viruses, between 80 and $150 \mathrm{~nm}$ in diameter, that contain two copies of a single-stranded 7-12 kb genome. ${ }^{1-4}$ Due to their limited genome, retroviruses require a host for successful replication. By integrating a double-stranded DNA copy of their viral genome into the host genome, they ensure replication along with the host genome during each cell cycle.

The cycle of viral replication of HIV-1 is depicted in Figure 1. Most of the steps of the viral replication cycle have been studied via ensemble biochemistry. The knowledge obtained from these investigations has been critical in the development of the viral inhibitors now available. However, it is generally accepted that only $0.1-10 \%$ of the virions binding to the cell surface eventually integrate their genome into the host. ${ }^{4}$ What makes a virus particle successfully integrate? Does a successful virus follow a different path within the cell? Which protein interactions determine productive infections? Understanding the molecular mechanisms behind viral infections, and how these affect cells, is critical for developing new treatments or vaccines. Single-virus and single-molecule analyses can reveal which different steps and host factors are required for a successful infection.
Fluorescent labeling of viral proteins and viral genome in living cells, combined with sensitive fluorescence microscopic techniques, provides an excellent approach for realizing a detailed and mechanistic understanding of the viral replication cycle and its underlying cell biology. ${ }^{5-7}$ In this Perspective, we demonstrate how imaging single virions can provide unique insights into the hidden life cycle of a retrovirus. Without being exhaustive, we give examples of single-virus-imaging applications in human immunodeficiency virus (HIV) research. In the final section, we discuss some of the key remaining questions in virology, specifically for HIV, for which single-particle imaging can help researchers find answers.

\section{CHALLENGES AND ADVANTAGES OF SINGLE-VIRUS IMAGING}

Fluorescent Labeling of Virus Particles. The first step toward imaging single viral particles is labeling the viral 


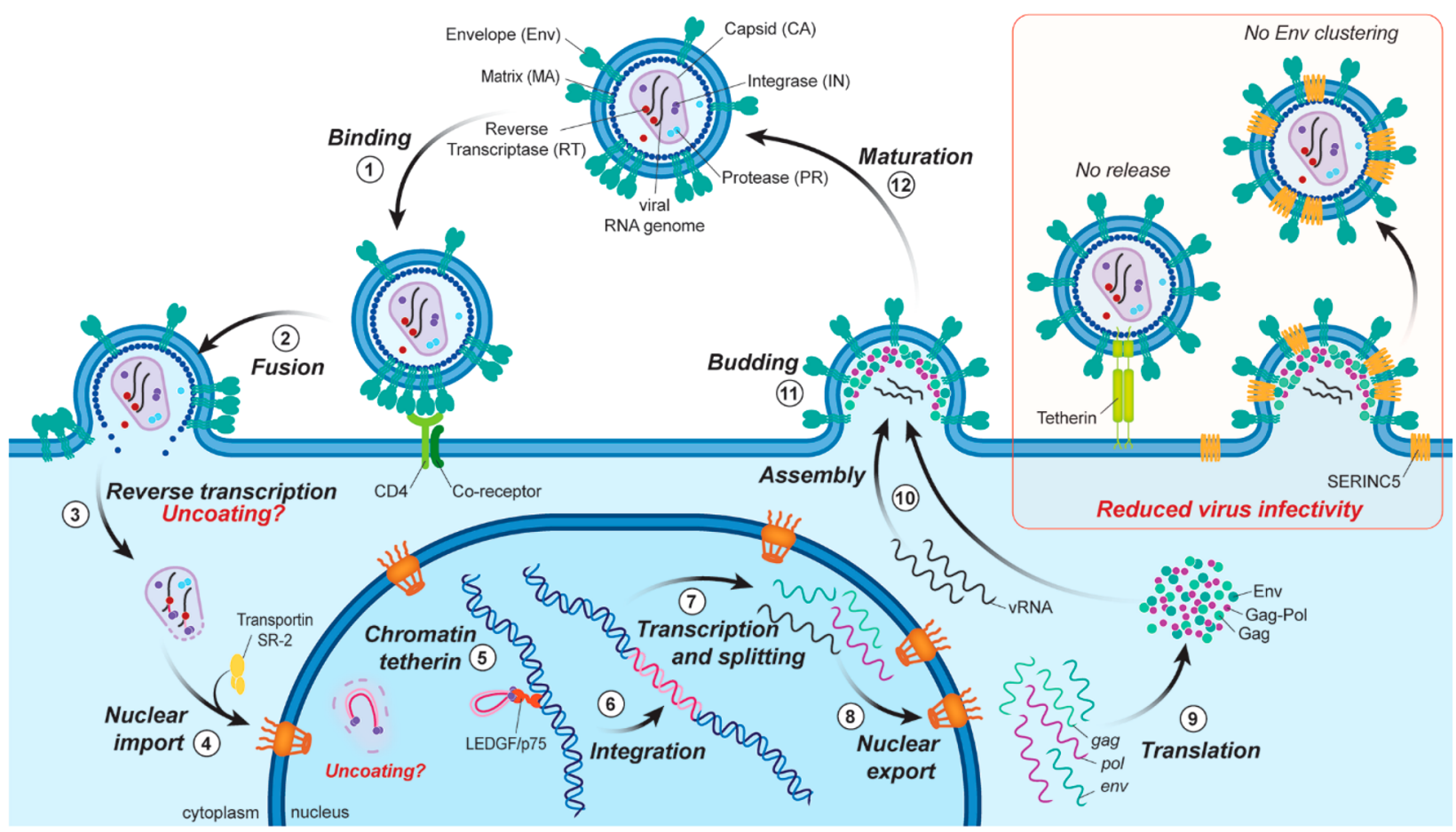

Figure 1. Schematic overview of the replication cycle of retroviruses. After envelope (Env)-mediated binding of the virus to the cell (1), fusion of the viral and cellular membrane is initiated (2), resulting in the release of the viral content in the cytoplasm. Next, reverse transcription of the viral RNA to a double-stranded DNA takes place (3). After completion of reverse transcription, the viral complex is referred to as the viral preintegration complex (PIC) and will be transported into the nucleus (with the involvement of transportin SR-2, step 4). Nuclear import is followed by binding of the lens-epithelium-derived growth factor (LEDGF), which is needed for tethering and targeting of the PIC to the host genome (5). After integration (6), the provirus constitutes an integral part of the genome and provides a lasting template for viral gene expression (7) and subsequent nuclear export (8) and translation in new proteins (9). Viral proteins and viral RNA will assemble at the plasma membrane (10), where new immature virions assemble and bud off the plasma membrane (11). Virus release can be restricted by Tetherin. Upon protease activation, Gag and Gag-pol proteins are cleaved and viruses mature (12). When serine incorporator 5 (SERINC5) is incorporated into the viral particles, Env clusters are not formed, reducing the infectivity of the produced virions.

components with high molecular specificity and efficiency. In addition, the labeling degree needs to be carefully adjusted: it needs to be high enough to enable sensitive detection but low enough not to influence functionality. Furthermore, the different imaging applications require fluorophores with distinct spectroscopic properties (molecular brightness, fluorescence lifetime, photostability, color, photoswitching capabilities, etc.). The choices of which molecule to label, how to label it, and which tag to use are linked to the biological question under investigation.

\section{The first step toward imaging single viral particles is labeling the viral components with high molecular spe- cificity and efficiency.}

The exposed components of viral particles, namely envelope proteins and the lipid bilayer, are readily available for labeling using synthetic, photostable fluorophores. ${ }^{8}$ A greater challenge is the labeling of internal components, which are typically inaccessible and often require permeabilization of the virus for chemical labeling.

Over the years, different strategies have been developed to label the viral genome of HIV-1. For example, intracellular mRNA can be directly visualized by fusing a fluorescent protein to an RNA-binding protein, which is expressed along with the target mRNA. ${ }^{9,10}$ Alternatively, during reverse transcription, ethynyl-functionalized deoxynucleoside triphosphates (dNTPs) are incorporated in the complementary DNA. The dNTPs enable covalent linkage with azide reactive fluorophores via a copper-catalyzed azide-alkyne cycloaddition, a technology that is commonly referred to as "click chemistry". Another approach relies on fluorescence in situ hybridization (FISH). This method exploits branched DNA technology to add a fluorescent probe to a specific nucleotide sequence and has been use to visualize either RNA (ViewRNA) $^{11,12}$ or DNA (ViewHIV) ${ }^{13}$ in virions.

For labeling viral proteins, a plethora of methods are available. The method of choice for most research groups is the insertion of either a gene encoding a fluorescent protein (FP) or a self-labeling protein tag (CLIP/SNAP/HaLo tag $)^{1,14}$ fused to the protein of interest. The large array of FPs available, including photoswitchable proteins, allied to perfect labeling specificity, make this approach attractive for (single) virus imaging. ${ }^{15,16}$ Like all retroviruses, the HIV-1 genome encodes three major open reading frames: group-specific antigen ( $g a g)$, polymerase ( $p o l)$, and envelope (env) polyproteins. The gag gene encodes for the structural proteins matrix, capsid (CA), nucleocapsid, and p6. The viral enzyme protease (PR), reverse transcriptase (RT), and integrase (IN) are encoded by the pol gene. Due to small genomes, open reading frame overlaps, and multifunctional proteins, incorporation of the coding sequence for a (fluorescent) protein into 
the viral genome is accompanied by some challenges. ${ }^{17}$ Consequently, most viral proteins cannot be labeled using this approach.

Another method to label viral proteins relies on the viral accessory protein viral protein $\mathrm{R}(\mathrm{Vpr})$, which is incorporated into viral particles through specific interactions with the $\mathrm{p} 6$ domain of the polyprotein Gag. The Vpr protein can be used to shuttle foreign proteins into HIV-1 particles. Fluorescentprotein-tagged versions of $\mathrm{Vpr}$ itself, as well as other viral proteins, have been successfully used to label the inner core of HIV-1 particles. ${ }^{18,19}$

Independent of the method chosen to incorporate a labeled protein into the viral particles, the tag itself might alter the protein's function and/or affect viral morphology and infectivity. When the introduction of a protein tag yields mature virions but with reduced infectivity, co-expression of modified and wild-type proteins can rescue virus infectivity (as shown for Gag). ${ }^{20-22}$ Alternatively, the use of smaller fluorescent tags can be advantageous. An example is the introduction of a short peptide tag containing a tetracysteine motif (e.g., Cys-Cys-Pro-Gly-Cys-Cys). This tag interacts with cell-permeable biarsenical dyes (FlAsH, ReAsH), thereby enabling it to label specifically the viral protein of interest. This approach has been used to image HIV-1 complexes in living cells. ${ }^{8,23-27}$

Another class of small labels for virus research is the biorthogonal click labels. ${ }^{17}$ Here, a noncanonical amino acid (ncAA) is built into the protein of interest at a specific site during viral synthesis, in amber-suppressed producer cells. During or after production, fluorescent labeling is carried out by adding an organic dye that is designed to react specifically with the ncAA. This method has been used to study the nanoscale distribution of HIV-1 Env protein on the surface of viral particles using stimulated emission depletion (STED) microscopy. ${ }^{28}$

Regardless of the fluorescent tag used, single-virus imaging will have to rely on quantitative biochemical/virology tools for functional characterization of labeled viral particles. The current trend is to label the minimum viral components needed with labels as small as possible and preferentially without interference with the replication steps of the virus.

Resolution at the Subviral and Molecular Levels. The diameter of HIV-1 is between 120 and $145 \mathrm{~nm} .^{2,29}$ The spatial resolution obtained with conventional fluorescence microscopy, however, is limited by the diffraction of the light to approximately 200-250 $\mathrm{nm}$. The development of superresolution fluorescence methods has made it possible to achieve lateral resolution down to $10-20 \mathrm{~nm} .{ }^{30}$ This improved resolution has opened the possibility to visualize structural features of individual viral particles and to define the subviral protein distribution under physiological conditions. ${ }^{6}$

The most commonly used techniques to overcome the diffraction resolution limit are STED microscopy and singlemolecule localization microscopy. These methods are briefly explained below (for a more complete review on superresolution fluorescence microscopy, see ref 30). Stimulated emission depletion microscopy is based on depleting the emission of fluorophores in the outer region of the diffraction limited spot with a red-shifted depletion laser pulse that is referred to as the STED beam. The selective deactivation of fluorophores through stimulated emission minimizes the effective fluorescent spot size. The increase in spatial resolution is dependent on the STED depletion intensity, and, typically, a resolution of $30-50 \mathrm{~nm}$ can be obtained. ${ }^{31,32}$ Single-molecule localization microscopy methods include photoactivated localization microscopy ${ }^{33}$ and (direct) stochastic optical reconstruction microscopy. ${ }^{34,35}$ These methods make use of fluorophores that can be converted in a stochastic manner from an "off" to an "on" state upon illumination by a certain wavelength. $^{36}$ Stochastic light-induced switching of the fluorophores enables temporal separation of spatially overlapping molecules. The localization of each emitter can be determined by fitting a two-dimensional (2D) Gaussian function to the point-spread function of the detected signal, with a precision of $20-30 \mathrm{~nm}$. Super-resolved images can be reconstructed from the calculated localizations. ${ }^{37}$ Over the years, these methods have been used to elucidate the protein organization in isolated viral particles and within infected cells. $6,27,28,38-41$

Examples of such studies probe the organization and dynamics of viral assembly sites. During virus assembly, the Gag and Gag-Pol proteins, together with two viral RNA strands, are recruited to the plasma membrane (Figure 1, step 10). There, Env molecules are incorporated and the endosomal sorting complexes required for transport (ESCRT) machinery enables budding of the virus. ${ }^{42}$ The nanoscale organization of different ESCRT components at HIV assembly sites was elucidated using three-dimensional (3D) super-resolution microscopy. ${ }^{43}$ Lippincott-Schwartz and co-workers showed that ESCRT subunits localized within the head of budding virions and that HIV release was triggered by the membrane association of ESCRT subunits.

Single-molecule localization microscopy has also been instrumental in resolving the spatial distribution of nascent Gag assemblies in relation to other viral or cellular proteins. Heilemann et al. have shown that Env glycoproteins are recruited to the viral budding sites in a ring-like formation, accumulating at the periphery rather than in the center of the budding sites. Env-rich membrane areas surrounding the budding sites were devoid of detectable Gag molecules, suggesting that Env proteins are recruited through Gaginduced alterations in the surrounding nascent viral bud. ${ }^{38}$ Combined biochemical and super-resolution microscopy have been used to investigate the cellular restriction factor tetherin. This molecule inhibits the release of HIV-1 through direct incorporation into viral membranes, a process that is counteracted by the HIV-1 protein vpu (viral protein unique). Lehmann et al. have shown that during viral assembly, extended tetherin dimers incorporate their $\mathrm{N}$-termini into the assembling virions and, in this way, restrict HIV-1 release (Figure 1). ${ }^{39}$

In other studies, STED has been used to investigate the distribution of Env molecules on the surface of individual HIV1 particles. ${ }^{28,40}$ Chojnacki et al. used dual-color STED to discover that membrane patches of the cellular receptor CD4 can induce clustering of mobile Env molecules on the viral particles, promoting efficient entry of the virus in the cell. ${ }^{40}$ This result was later confirmed using ncAA labeling of Env proteins. ${ }^{28}$ The authors suggested that Env trimers are initially recruited to viral budding sites in a random distribution, and, after proteolytic maturation, they coalesce into a single Env focus to enhance the interaction with a CD4 patch on the target cell surface. The high mobility and clustering of Env proteins appears to be crucial for successful infection. Consequently, molecules disturbing Env cluster formation will reduce the infectivity of the viral particles. 
One of these molecules is serine incorporator protein 5 (SERINC5). In this issue of ACS Nano, Melikyan and coworkers describe using $2 \mathrm{D}$ and $3 \mathrm{D}$ super-resolution microscopy to investigate the effect of SERINC5 on the distribution of Env proteins in single HIV-1 particles. ${ }^{41}$ SERINC5 is a cellular restriction factor that can be incorporated into the progeny virus. It is known that SERINC5 incorporation into viral particles alters the Env structure, inhibiting the Envmediated virus fusion with the target cell and, consequently, reducing HIV-1 infectivity. Similar to previous studies, the authors found that Env proteins form clusters on the surface of the viral particles. Moreover, SERINC5 was shown to disrupt the Env clusters, but SERINC2, which lacks antiviral activity, did not. Poor colocalization of Env and SERINC5 molecules on the viral membrane implies an indirect mechanism of SERINC5-mediated inhibition of HIV-fusion (Figure 1). This work is a beautiful illustration of how single-molecule/particle and super-resolution studies can provide mechanistic insights into viral biology.

\section{In this issue of ACS Nano, Melikyan and co-workers describe using two-dimen- sional and three-dimensional super- resolution microscopy to investigate the effect of SERINC5 on the distribu- tion of envelope proteins in single HIV-1 particles.}

Optical techniques are pushing toward submolecular resolution. An excellent example is single-molecule Förster resonance energy transfer (smFRET). ${ }^{44-47}$ Förster resonance energy transfer is the phenomenon of radiationless energy transfer between a suitable pair of fluorophores (donor, acceptor) in close proximity. ${ }^{48}$ This method is highly sensitive to the distance between two chromophores in the range of 2$10 \mathrm{~nm}$, making it extraordinarily suitable to investigate molecular conformations and interactions. ${ }^{49}$ When acceptor and donor fluorophores are placed in different locations on the same molecule, smFRET can be used to quantify protein structures and conformational dynamics in real time with Ångstrom precision. An example of smFRET in HIV research is the imaging of HIV-1 Env trimer conformational dynamics. ${ }^{50}$ The analysis of Förster resonance energy transfer (FRET) signals revealed the molecular events that underlie the two-step activation of HIV-1 Env by CD4 and coreceptor. Undoubtedly, smFRET might provide unique insights into other steps in the viral life cycle. The true challenge and relevance for single-virus research lies in its application to studying intracellular events.

Live Imaging of the Viral Replication Cycle. Only by tracking single viruses from cellular uptake through integration can we understand the different steps and host factors that are required for productive infection. Advances in labeling strategies and fluorescence microscopy methods have enabled imaging viruses within living cells in real time. ${ }^{51-53}$

MacDonald et al. used eGFP-Vpr-labeled HIV-1 virus to visualize early post-entry events. ${ }^{54}$ Tracking of single viral particles revealed that after entering the cytoplasm, HIV-1 uses cytoplasmic dynein and the microtubule network to migrate toward the nucleus. Later, FlAsH labeling of IN enabled the sensitive detection and tracking of viral complexes in both the cytoplasm and nucleus. ${ }^{24}$ Although the cytoplasmic HIV-1 complexes showed fast microtubule-directed movements toward the nucleus, intranuclear HIV-1 complexes showed only restrained diffusive movement within the nucleus, which could be indicative of interactions with chromatin.

For productive infection of host cells, the viral capsid must be disassembled in a process referred to as uncoating. Debate on the exact mechanism, timing, and localization of uncoating is ongoing. ${ }^{55}$ The large size of the virus core (61 nm width, 120 $\mathrm{nm}$ length) in comparison to the nuclear pore $(40 \mathrm{~nm})$ requires active uncoating before nuclear import. ${ }^{56}$ Using timelapse imaging, Francis et al. showed that docking and uncoating of $\mathrm{HIV}-1$ at the nuclear envelope are a prerequisite for nuclear import. ${ }^{53,57}$ At 90 min post-infection and $35 \mathrm{~min}$ after docking to the nuclear envelope, the amount of CA in the viral particles decreased, which is indicative of uncoating. ${ }^{57} \mathrm{~A}$ similar finding was obtained by Mamede et al., who found that cytoplasmic uncoating of HIV-1 particles occurred within 90 min post-infection. ${ }^{55}$ Recently Burdick et al. published a controversial hypothesis on uncoating and reverse transcription of HIV-1 particles in the nucleus, close to their integration site. ${ }^{20}$ After docking at the nuclear envelope for $1.9 \mathrm{~h}, \mathrm{GFP}-\mathrm{CA}$-labeled viral complexes were imported into the nucleus within $\sim 4.4 \mathrm{~h}$ post-infection. Importantly, although the exact location of the uncoating process remains under debate, several groups have shown that the number of CA moieties in nuclear complexes is lower than in those found in the cytoplasm. ${ }^{21,27,58}$

Despite the potential of fluorescence microscopy to investigate the molecular mechanisms underlying viral infection, its application in live imaging of a single virus remains limited. The photon yield of commonly used fluorophores limits the total number of image frames that can be recorded before photobleaching occurs. In addition, the field of view that can be recorded with a high acquisition rate limits the number of particles that can be imaged simultaneously. Strategies to improve fluorophore photon yields and a continued transition to automated high-content imaging are, therefore, most promising in popularizing timelapse single-virus imaging.

Quantifying Interactions between Viral and Host Proteins. Throughout the replication cycle, retroviruses depend on the host cell, from which they borrow functionalities such as active nuclear import (e.g., Christ et al. $)^{19}$ or chromatin tethering (e.g., Hendrix et al.). ${ }^{59}$ Virus replication relies on specific interactions of viral proteins with themselves (oligomerization), with other proteins from the virus, or with proteins present in the host cell. The most popular methods for studying molecular interactions in cells are fluorescence fluctuation spectroscopy (FFS), and FRET.

Fluorescence fluctuation spectroscopy is a powerful quantitative spectroscopic technique used to analyze the mobility and concentration of molecules and, eventually, their interactions with other molecules. ${ }^{60,61}$ Imaging variants of FFS methods include raster image correlation spectroscopy $(\mathrm{RICS})^{62-66}$ and number and brightness (N\&B) analysis. ${ }^{67}$ In RICS, a raster pattern is scanned by the laser on a confocal laser scanning microscope, creating a space-time matrix of pixels within the image. The temporal information is included in each image as the obtained pixels are collected over time. From the spatial correlation function, the diffusion coefficient and the binding processes can be identified. ${ }^{68,69}$ In contrast, $\mathrm{N} \& \mathrm{~B}$ analysis is based on the intensity fluctuations present in 
the individual pixels over the course of the recorded image series. The effective molecular brightness and number of molecules for a single diffusing species are determined from the average value and variance of the fluorescence signal. ${ }^{67}$

An FFS analysis of single virions revealed that the HIV-1 Gag stoichiometry varies between 750 and 2500 per virion, suggesting that the amount of Gag incorporated in single viral particles depends on the expression of the Gag protein in the cell. ${ }^{70}$ Fogarty et al. have shown that the oligomeric state of cytosolic Gag also depends on the concentration of this protein during viral assembly. ${ }^{71}$ A more recent investigation showed that a fraction of the cytosolic Gag partly diffuses as a monomer, frequently interacting with RNA, while another fraction diffuses much slower, as an oligomer. The latter species presumably functions as a seed for new assembly sites at the plasma membrane. ${ }^{72}$ Derdowski et al. used FRET to demonstrate that most of the interactions between Gag proteins take place at the cellular membrane. The authors also showed that Gag multimerization occurred at multiple distinct sites at the plasma membrane. ${ }^{73}$

Borrenberghs et al. used FRET to probe the relative oligomeric state of HIV-1 IN inside single viral particles. $^{74}$ Small-molecule inhibitors of the interaction between HIV-IN and the host factor lens-epithelium-derived growth factor (LEDGF)/p75, referred to as LEDGINs, were shown to stimulate IN oligomerization in the budding virus. ${ }^{74,75}$ Increased IN oligomerization resulted in crippled virus with aberrant electron microscopy morphology. Similar labeling and FRET analysis of HIV-1 IN were later used to reveal how LEDGF/p75 alters IN stoichiometry upon nuclear import. ${ }^{58} \mathrm{~A}$ similar mechanism was also observed for the IN from Moloney murine leukemia virus upon interaction with bromo- and extraterminal domain proteins. ${ }^{76}$

In general, during the retroviral replication cycle, multiple different components must interact with one another in a tightly time-space-orchestrated manner. Fluorescence microscopy provides the means for quantifying all these interactions simultaneously and quantitatively. However, specific applications in single-virus research are scarce. With the ability to record spectrally resolved microscopy images and videos, or even multiparametric data, it will be possible to unmix multiple fluorophores and, thus, to monitor the fate of different proteins simultaneously, disentangling intricate relations between different labeled viral and host components during the viral replication cycle. The real challenge lies in the analysis tools to convert such multidimensional fluorescence data to meaningful insights on viral biology.

\section{REMAINING QUESTIONS IN HIV VIROLOGY}

As shown in the previous section, imaging of single-viral particles has already facilitated answering many biological questions concerning the retroviral replication cycle. However, much remains unknown.

How Many Viral Particles Are Required To Infect a Cell Productively? This is perhaps one of the most intriguing questions in virology. The multiplicity of infection (MOI) is a term used in microbiology that refers to the ratio of agents (e.g., virus, bacteria, phage) to infection targets (e.g., cell). For example, when referring to a group of cells inoculated with virus particles, the MOI is the ratio of the number of virus particles to the number of target cells present. However, a MOI of 1 (one virus per cell) does not mean that all cells will be infected by one particle. For a specific MOI, the number of particles that a cell receives is described by a Poisson distribution. Accordingly, at a MOI of 1 , only $63 \%$ of cells will contain viral particles. To detect particles in more than $95 \%$ of the cells, a minimum MOI of 3 is required. Even then, not all cells that contain particles will be infected. Biologically speaking, it is extremely unlikely that one internalized virus particle will be able to yield one infection.

A more accurate way to determine how many viral particles are necessary to infect a cell is to measure the viral titer. Functional titers measure infectivity: one infectious unit represents the number of viruses required to produce a cytopathogenic effect (e.g., a giant cell) or reporter read out in one cell (e.g., eGFP expression). Physical titers measure how much virus is present and can be obtained by measuring protein ( $\mathrm{p} 24)$ or RNA content or by electron microscopy (particle count). Interestingly, from comparisons of functional titers with physical titers, it appears that only $0.1-10 \%$ ( 1 in 1000 to 1 in 10) of HIV-1 particles present during infection of cells yield an integrated provirus. ${ }^{4}$

The low efficiency of viral infection is thought to be intrinsically linked to the high level of heterogeneity present between viral particles. The virus assembled in host cells may not all be functionally competent. This finding is true for a virus produced in vitro, using cell culture methods, but a less than optimal production efficiency is also likely to happen in vivo. In a way, virus infection can be seen as 'the survival of the fittest'. Infection of cells is all about overcoming barriers: physical barriers such as the cellular and nuclear membranes, and cellular protection mechanisms such as restriction factors. Both barriers may inherently also select the fittest particles, particles that can cross membranes and nucleopores and overcome restriction factors. From an evolutionary perspective, redundancy may help selection if infection occurs with particles that possess different genomes. Another plausible justification for the observed low MOI is that the excess of viral particles entering the cell, even the nonfunctional particles, may work as decoys against innate immunity/restriction factors.

Single-virus imaging and live tracking can be used to pinpoint the viral "superheroes", particles that cross membranes, avoid restriction factors, and result in productive integration. Retro-tracking will identify the characteristics of these particles to discriminate them from non-integrating particles. Live imaging will reveal time-dependent changes in functionality (i.e., how a virus that arrives earlier may restrict the infection by late comers or rather prepare their efficient integration). ${ }^{77,78}$ The examples discussed in this Perspective highlight the potential of single-virus imaging to address some of the open questions in virology.

\section{Single-virus imaging and live tracking can be used to pinpoint the viral "superheroes", particles that cross membranes, avoid restriction factors, and result in productive integration.}

Do (All) Particles in the Cytoplasm Retro-Transcribe? Not all particles enter the cell, and not all particles present in the cytoplasm enter the nucleus. Recently, there are even doubts on whether reverse transcription of HIV-1 occurs in the cytoplasm or in the nucleus. ${ }^{20}$ Inefficient labeling of reverse 
transcription in cytoplasmic PICs may be linked to variable degrees of reverse transcription. ${ }^{79,80}$ Is reverse transcription associated with uncoating? Fluorescent capsid labeling and live-cell imaging should provide answers to these questions. $^{20,21}$

How Are Pre-Integration Complexes Selected To Enter the Nucleus? The integrase content of nuclear particles is lower than that of particles in the cytoplasm, implying morphological alterations at the nucleopore. ${ }^{27,58}$ It is not yet known whether only particles with limited IN content pass the nucleopore or if all particles need to be trimmed at the nucleopore from excess IN. Answering this question will require live imaging and careful analysis of the amount of CA present during nuclear import. The host factors required to transport the PIC across the nuclear membrane are also a matter of debate. Super-resolution microscopy and FRET should provide sufficient resolution to identify the authentic protein-protein interactions.

Are All Integrants Transcriptionally Active? From the particles that enter the nucleus, it is not known how many integrate into the genome of the host. We do know that not all proviruses are transcriptionally active. HIV evolved to yield both productive and latent infections. Productive infections ensure spreading within the host and to other hosts. Latent infections ensure long-term survival of the virus in the host. The chromatin environment of the provirus apparently determines transcriptional activity. ${ }^{81,82}$ LEDGF/p75 plays an important role by selecting transcriptionally active regions of the genome through recognition of the $\mathrm{H} 3 \mathrm{~K} 36 \mathrm{me} 3$ epigenetic mark. DNA imaging of viral DNA and mRNA in the same cell can be used to discriminate latent and productive infections.

How is Gag-Pol Involved in the Onset of Assembly? $\mathrm{HIV}-1$ assembly is controlled primarily by the Gag polyprotein. Gag orchestrates HIV-1 assembly by recruiting all the building blocks required for the formation of fully infectious viral particles and the synthesis of the Gag precursor protein alone is sufficient to produce virus-like particles. However, because the viral enzymes PR, RT, and IN are produced from the GagPol polyprotein, its incorporation into virions is required for infectivity. ${ }^{83}$ This incorporation is likely to occur via "coassembly" with Gag. ${ }^{84}$ During and after release of virions from cells, the Gag precursor protein is cleaved by PR into mature proteins. Because dimerization of $\mathrm{PR}$ is essential for $\mathrm{PR}$ activity, oligomerization of the Gag-Pol polyprotein is a prerequisite for the formation of mature virions. Does GagPol form oligomers because of IN dimerization? When are these oligomers formed? Are the changes in the Gag-Pol oligomerization induced by LEDGINs responsible for the observed morphological defects of the virus ${ }^{75}$ These questions could be addressed with quantitative FFS and super-resolution microscopy.

Exciting technological advances in the field of fluorescence microscopy, including super-resolution, more advanced fluctuation imaging methods, and innovative labeling strategies, have opened windows that will yield detailed insights into the viral replication cycle. Although we described examples from the HIV field, the methods presented here have been applied to other viruses as well. For instance, live-cell imaging has revealed that the simian virus 40 utilizes endocytosis through caveolae for infectious entry into host cells ${ }^{85}$ and that the Ebola virus is internalized via micropinocytosis. ${ }^{86}$ More recently, fluorescently labeled viral ribonucleoprotein complexes were used to track the uncoating of single influenza A viruses in living cells. ${ }^{87}$ These viruses, responsible for some of the epidemics that occurred in the 20th century, have been around for decades. Yet, imaging of single viral particles is still unravelling new information concerning their modus operandi. The methods are well established and can be readily adapted and applied to investigate other, new viruses. To the best of our knowledge, at the date of publication of this Perspective, there are no records of single-virus imaging of SARS-CoV-2. However, we believe that single-virus-imaging approaches will be of utmost importance for understanding the molecular mechanisms involved in the infection caused by SARS-CoV-2 and the subsequent development of new treatments or vaccines.

\section{AUTHOR INFORMATION}

\section{Corresponding Authors}

Susana Rocha - Molecular Imaging and Photonics, Chemistry Department, KU Leuven, B-3001 Heverlee, Flanders, Belgium; 다. orcid.org/0000-0003-1258-9396; Email: susana.rocha@ kuleuven.be

Zeger Debyser - Laboratory for Molecular Virology and Gene Therapy, Department of Pharmaceutical and Pharmacological Sciences, KU Leuven, B-3001 Heverlee, Flanders, Belgium; Email: zeger.debyser@kuleuven.be

\section{Authors}

Jelle Hendrix - Molecular Imaging and Photonics, Chemistry Department, KU Leuven, B-3001 Heverlee, Flanders, Belgium; Dynamic Bioimaging Lab, Advanced Optical Microscopy Centre and Biomedical Research Institute (BIOMED), Hasselt University, B-3590 Diepenbeek, Flanders, Belgium; (1) orcid.org/0000-0001-5731-1297

Doortje Borrenberghs - Molecular Imaging and Photonics, Chemistry Department, KU Leuven, B-3001 Heverlee, Flanders, Belgium

Johan Hofkens - Molecular Imaging and Photonics, Chemistry Department, KU Leuven, B-3001 Heverlee, Flanders, Belgium; Max Planck Institute for Polymer Research, 55128 Mainz, Germany; ○ orcid.org/0000-0002-9101-0567

Complete contact information is available at:

https://pubs.acs.org/10.1021/acsnano.0c06369

\section{Notes}

The authors declare no competing financial interest.

\section{ACKNOWLEDGMENTS}

The authors acknowledge the funding from the Research Foundation - Flanders (FWO, G0A817N, G0A5316N, $1529418 \mathrm{~N}$, SBO-Saphir) and by KU Leuven Research Council (C14/16/053, C14/17/095-3M170311). J.H. acknowledges support from the Flemish government through long term structural funding Methusalem (CASAS2, Meth/15/04).

\section{ABBREVIATIONS}

CA, capsid; CD 4, cluster of differentiation 4; DNA, DNA; eGFP, enhanced green fluorescent protein; Env, envelope; ESCRT, endosomal sorting complexes required for transport; FFS, fluorescence fluctuation spectroscopy; FLIM, fluorescence lifetime imaging microscopy; FP, fluorescent protein; FRET, Förster resonance energy transfer; Gag, group-specific antigen; HIV, human immunodeficiency virus; IN, integrase; LEDGF, lens-epithelium-derived growth factor; LEDGIN, 
LEDGF/p75-IN interaction inhibitor; MOI, multiplicity of infection; N\&B, number and brightness; NPC, nuclear pore complex; PIC, preintegration complex; PIE, pulsed interleaved excitation; PR, protease; RICS, raster image correlation spectroscopy; RNA, ribonucleic acid; ROI, region of interest; $\mathrm{RT}$, reverse transcriptase; SERINC5, serine incorporator 5; STED, stimulated emission depletion microscopy; Vpr, viral protein $\mathrm{R}$

\section{REFERENCES}

(1) Eckhardt, M.; Anders, M.; Muranyi, W.; Heilemann, M.; KrijnseLocker, J.; Müller, B. A SNAP-Tagged Derivative of HIV-1-A Versatile Tool To Study Virus-Cell Interactions. PLoS One 2011, 6, No. e22007.

(2) Briggs, J. A. G.; Johnson, M. C.; Simon, M. N.; Fuller, S. D.; Vogt, V. M. Cryo-Electron Microscopy Reveals Conserved and Divergent Features of Gag Packing in Immature Particles of Rous Sarcoma Virus and Human Immunodeficiency Virus. J. Mol. Biol. 2006, 355, 157-168.

(3) Yeager, M.; Wilson-Kubalek, E. M.; Weiner, S. G.; Brown, P. O.; Rein, A. Supramolecular Organization of Immature and Mature Murine Leukemia Virus Revealed by Electron Cryo-Microscopy: Implications for Retroviral Assembly Mechanisms. Proc. Natl. Acad. Sci. U. S. A. 1998, 95, 7299-7304.

(4) Coffin, J. M.; Hughes, S. H.; Varmus, H. E. The Interactions of Retroviruses and Their Hosts. In Retroviruses; Coffin, J. M., Hughes, S. H., Varmus, H. E., Eds.; Cold Spring Harbor Laboratory Press: Cold Spring Harbor, NY, 1997.

(5) Brandenburg, B.; Zhuang, X. Virus Trafficking-Learning from Single-Virus Tracking. Nat. Rev. Microbiol. 2007, 5, 197-208.

(6) Müller, B.; Heilemann, M. Shedding New Light on Viruses: Super-Resolution Microscopy for Studying Human Immunodeficiency Virus. Trends Microbiol. 2013, 21, 522-533.

(7) Smith, D. E. Single-Molecule Studies of Viral DNA Packaging. Curr. Opin. Virol. 2011, 1, 134-141.

(8) Wojta-Stremayr, D.; Pickl, W. F. Fluorosomes: Fluorescent Virus-Like Nanoparticles that Represent a Convenient Tool To Visualize Receptor-Ligand Interactions. Sensors 2013, 13, 87228749 .

(9) Jouvenet, N.; Simon, S. M.; Bieniasz, P. D. Imaging the Interaction of HIV-1 Genomes and Gag during Assembly of Individual Viral Particles. Proc. Natl. Acad. Sci. U. S. A. 2009, 106, 19114-19119.

(10) Fusco, D.; Accornero, N.; Lavoie, B.; Shenoy, S. M.; Blanchard, J.-M.; Singer, R. H.; Bertrand, E. Single MRNA Molecules Demonstrate Probabilistic Movement in Living Mammalian Cells. Curr. Biol. 2003, 13, 161-167.

(11) Feeley, E. M.; Sims, J. S.; John, S. P.; Chin, C. R.; Pertel, T.; Chen, L.-M.; Gaiha, G. D.; Ryan, B. J.; Donis, R. O.; Elledge, S. J.; Brass, A. L. IFITM3 Inhibits Influenza A Virus Infection by Preventing Cytosolic Entry. PLoS Pathog. 2011, 7, No. e1002337.

(12) Yang, W.; Maqsodi, B.; Ma, Y.; Bui, S.; Crawford, K. L.; McMaster, G. K.; Witney, F.; Luo, Y. Direct Quantification of Gene Expression in Homogenates of Formalin-Fixed, Paraffin-Embedded Tissues. BioTechniques 2006, 40, 481-486.

(13) Chin, C. R.; Perreira, J. M.; Savidis, G.; Portmann, J. M.; Aker, A. M.; Feeley, E. M.; Smith, M. C.; Brass, A. L. Direct Visualization of HIV-1 Replication Intermediates Shows that Capsid and CPSF6Modulate HIV-1 Intra-Nuclear Invasion and Integration. Cell Rep. 2015, 13, 1717-1731.

(14) Keppler, A.; Pick, H.; Arrivoli, C.; Vogel, H.; Johnsson, K. Labeling of Fusion Proteins with Synthetic Fluorophores in Live Cells. Proc. Natl. Acad. Sci. U. S. A. 2004, 101, 9955-9959.

(15) Rocha, S.; De Keersmaecker, H.; Uji-i, H.; Hofkens, J.; Mizuno, H. Photoswitchable Fluorescent Proteins for Superresolution Fluorescence Microscopy Circumventing the Diffraction Limit of Light. In Fluorescence Spectroscopy and Microscopy: Methods and
Protocols; Engelborghs, Y., Visser, A. J. W. G., Eds.; Methods in Molecular Biology; Humana Press: Totowa, NJ, 2014; pp 793-812. (16) Giepmans, B. N. G.; Adams, S. R.; Ellisman, M. H.; Tsien, R. Y. The Fluorescent Toolbox for Assessing Protein Location and Function. Science 2006, 312, 217-224.

(17) Sakin, V.; Paci, G.; Lemke, E. A.; Müller, B. Labeling of Virus Components for Advanced, Quantitative Imaging Analyses. FEBS Lett. 2016, 590, 1896-1914.

(18) Francis, A. C.; Di Primio, C.; Quercioli, V.; Boll, A.; Arosio, D.; Cereseto, A. A Second Generation HIV-IN-EGFP Fluorescent Viral System To Analyze HIV-1 in the Nuclear Compartment of Infected Cells. Retrovirology 2013, 10, P31.

(19) Christ, F.; Thys, W.; De Rijck, J.; Gijsbers, R.; Albanese, A.; Arosio, D.; Emiliani, S.; Rain, J.-C.; Benarous, R.; Cereseto, A.; Debyser, Z. Transportin-SR2 Imports HIV into the Nucleus. Curr. Biol. 2008, 18, 1192-1202.

(20) Burdick, R. C.; Li, C.; Munshi, M.; Rawson, J. M. O.; Nagashima, K.; Hu, W.-S.; Pathak, V. K. HIV-1 Uncoats in the Nucleus Near Sites of Integration. Proc. Natl. Acad. Sci. U. S. A. 2020, 117, 5486-5493.

(21) Zurnic Bonisch, I.; Dirix, L.; Lemmens, V.; Borrenberghs, D.; De Wit, F.; Vernaillen, F.; Rocha, S.; Christ, F.; Hendrix, J.; Hofkens, J.; Debyser, Z. Capsid-Labelled HIV To Investigate the Role of Capsid during Nuclear Import and Integration. J. Virol. 2020, 94, e01024.

(22) Müller, B.; Daecke, J.; Fackler, O. T.; Dittmar, M. T.; Zentgraf, H.; Kräusslich, H.-G. Construction and Characterization of a Fluorescently Labeled Infectious Human Immunodeficiency Virus Type 1 Derivative. J. Virol. 2004, 78, 10803-10813.

(23) Pereira, C. F.; Ellenberg, P. C.; Jones, K. L.; Fernandez, T. L.; Smyth, R. P.; Hawkes, D. J.; Hijnen, M.; Vivet-Boudou, V.; Marquet, R.; Johnson, I.; Mak, J. Labeling of Multiple HIV-1 Proteins with the Biarsenical-Tetracysteine System. PLoS One 2011, 6, No. e17016.

(24) Arhel, N.; Genovesio, A.; Kim, K.-A.; Miko, S.; Perret, E.; Olivo-Marin, J.-C.; Shorte, S.; Charneau, P. Quantitative FourDimensional Tracking of Cytoplasmic and Nuclear HIV-1 Complexes. Nat. Methods 2006, 3, 817-824.

(25) Whitt, M. A.; Mire, C. E. Utilization of Fluorescently-Labeled Tetracysteine-Tagged Proteins To Study Virus Entry by Live Cell Microscopy. Methods 2011, 55, 127-136.

(26) Rudner, L.; Nydegger, S.; Coren, L. V.; Nagashima, K.; Thali, M.; Ott, D. E. Dynamic Fluorescent Imaging of Human Immunodeficiency Virus Type $1 \mathrm{Gag}$ in Live Cells by Biarsenical Labeling. J. Virol. 2005, 79, 4055-4065.

(27) Lelek, M.; Di Nunzio, F.; Henriques, R.; Charneau, P.; Arhel, N.; Zimmer, C. Superresolution Imaging of HIV in Infected Cells with FlAsH-PALM. Proc. Natl. Acad. Sci. U. S. A. 2012, 109, 85648569

(28) Sakin, V.; Hanne, J.; Dunder, J.; Anders-Össwein, M.; Laketa, V.; Nikić, I.; Kräusslich, H.-G.; Lemke, E. A.; Müller, B. A Versatile Tool for Live-Cell Imaging and Super-Resolution Nanoscopy Studies of HIV-1 Env Distribution and Mobility. Cell Chem. Biol. 2017, 24, $635-645$.

(29) Wright, E. R.; Schooler, J. B.; Ding, H. J.; Kieffer, C.; Fillmore, C.; Sundquist, W. I.; Jensen, G. J. Electron Cryotomography of Immature HIV-1 Virions Reveals the Structure of the CA and SP1 Gag Shells. EMBO J. 2007, 26, 2218-2226.

(30) Vangindertael, J.; Camacho, R.; Sempels, W.; Mizuno, H.; Dedecker, P.; Janssen, K. P. F. An Introduction to Optical SuperResolution Microscopy for the Adventurous Biologist. Methods Appl. Fluoresc. 2018, 6, 022003.

(31) Hell, S. W.; Wichmann, J. Breaking the Diffraction Resolution Limit by Stimulated Emission: Stimulated-Emission-Depletion Fluorescence Microscopy. Opt. Lett. 1994, 19, 780-782.

(32) Klar, T. A.; Hell, S. W. Subdiffraction Resolution in Far-Field Fluorescence Microscopy. Opt. Lett. 1999, 24, 954-956.

(33) Betzig, E.; Patterson, G. H.; Sougrat, R.; Lindwasser, O. W.; Olenych, S.; Bonifacino, J. S.; Davidson, M. W.; Lippincott-Schwartz, 
J.; Hess, H. F. Imaging Intracellular Fluorescent Proteins at Nanometer Resolution. Science 2006, 313, 1642-1645.

(34) Heilemann, M.; van de Linde, S.; Schüttpelz, M.; Kasper, R.; Seefeldt, B.; Mukherjee, A.; Tinnefeld, P.; Sauer, M. SubdiffractionResolution Fluorescence Imaging with Conventional Fluorescent Probes. Angew. Chem., Int. Ed. 2008, 47, 6172-6176.

(35) Rust, M. J.; Bates, M.; Zhuang, X. Sub-Diffraction-Limit Imaging by Stochastic Optical Reconstruction Microscopy (STORM). Nat. Methods 2006, 3, 793-796.

(36) Heilemann, M.; Dedecker, P.; Hofkens, J.; Sauer, M. Photoswitches: Key Molecules for Subdiffraction-Resolution Fluorescence Imaging and Molecular Quantification. Laser Photonics Rev. 2009, 3, 180-202.

(37) Schmidt, T.; Schütz, G. J.; Baumgartner, W.; Gruber, H. J.; Schindler, H. Imaging of Single Molecule Diffusion. Proc. Natl. Acad. Sci. U. S. A. 1996, 93, 2926-2929.

(38) Muranyi, W.; Malkusch, S.; Müller, B.; Heilemann, M.; Kräusslich, H.-G. Super-Resolution Microscopy Reveals Specific Recruitment of HIV-1 Envelope Proteins to Viral Assembly Sites Dependent on the Envelope C-Terminal Tail. PLoS Pathog. 2013, 9, No. e1003198.

(39) Lehmann, M.; Rocha, S.; Mangeat, B.; Blanchet, F.; Uji-i, H.; Hofkens, J.; Piguet, V. Quantitative Multicolor Super-Resolution Microscopy Reveals Tetherin HIV-1 Interaction. PLoS Pathog. 2011, 7, No. e1002456.

(40) Chojnacki, J.; Staudt, T.; Glass, B.; Bingen, P.; Engelhardt, J.; Anders, M.; Schneider, J.; Müller, B.; Hell, S. W.; Kräusslich, H.-G. Maturation-Dependent HIV-1 Surface Protein Redistribution Revealed by Fluorescence Nanoscopy. Science 2012, 338, 524-528.

(41) Chen, Y.-C.; Sood, C.; Marin, M.; Aaron, J.; Gratton, E.; Salaita, K.; Melikyan, G. B. Super-Resolution Fluorescence Imaging Reveals That Serine Incorporator Protein 5 Inhibits Human Immunodeficiency Virus Fusion by Disrupting Envelope Glycoprotein Clusters. ACS Nano 2020, DOI: 10.1021/acsnano.0c02699.

(42) Freed, E. O. HIV-1 Assembly, Release and Maturation. Nat. Rev. Microbiol. 2015, 13, 484-496.

(43) Van Engelenburg, S. B.; Shtengel, G.; Sengupta, P.; Waki, K.; Jarnik, M.; Ablan, S. D.; Freed, E. O.; Hess, H. F.; LippincottSchwartz, J. Distribution of ESCRT Machinery at HIV Assembly Sites Reveals Virus Scaffolding of ESCRT Subunits. Science 2014, 343, 653-656.

(44) Hellenkamp, B.; Schmid, S.; Doroshenko, O.; Opanasyuk, O.; Kuehnemuth, R.; Adariani, S. R.; Ambrose, B.; Aznauryan, M.; Barth, A.; Birkedal, V.; Bowen, M. E.; Chen, H.; Cordes, T.; Eilert, T.; Fijen, C.; Gebhardt, C.; Goetz, M.; Gouridis, G.; Gratton, E.; Ha, T.; et al. Precision and Accuracy of Single-Molecule FRET Measurements-A Multi-Laboratory Benchmark Study. Nat. Methods 2018, 15, 669676.

(45) Kalinin, S.; Peulen, T.; Sindbert, S.; Rothwell, P. J.; Berger, S.; Restle, T.; Goody, R. S.; Gohlke, H.; Seidel, C. A. M. A Toolkit and Benchmark Study for FRET-Restrained High-Precision Structural Modeling. Nat. Methods 2012, 9, 1218-1225.

(46) Deniz, A. A.; Dahan, M.; Grunwell, J. R.; Ha, T. J.; Faulhaber, A. E.; Chemla, D. S.; Weiss, S.; Schultz, P. G. Single-Pair Fluorescence Resonance Energy Transfer on Freely Diffusing Molecules: Observation of Forster Distance Dependence and Subpopulations. Proc. Natl. Acad. Sci. U. S. A. 1999, 96, 3670-3675.

(47) Lerner, E.; Cordes, T.; Ingargiola, A.; Alhadid, Y.; Chung, S.; Michalet, X.; Weiss, S. Toward Dynamic Structural Biology: Two Decades of Single-Molecule Forster Resonance Energy Transfer. Science 2018, 359, No. eaan1133.

(48) Jares-Erijman, E. A.; Jovin, T. M. FRET Imaging. Nat. Biotechnol. 2003, 21, 1387-1395.

(49) Stryer, L.; Haugland, R. P. Energy Transfer: A Spectroscopic Ruler. Proc. Natl. Acad. Sci. U. S. A. 1967, 58, 719-726.

(50) Munro, J. B.; Gorman, J.; Ma, X.; Zhou, Z.; Arthos, J.; Burton, D. R.; Koff, W. C.; Courter, J. R.; Smith, A. B.; Kwong, P. D.; Blanchard, S. C.; Mothes, W. Conformational Dynamics of Single
HIV-1 Envelope Trimers on the Surface of Native Virions. Science 2014, 346, 759-763.

(51) Seisenberger, G.; Ried, M. U.; Endreß, T.; Büning, H.; Hallek, M.; Bräuchle, C. Real-Time Single-Molecule Imaging of the Infection Pathway of an Adeno-Associated Virus. Science 2001, 294, 19291932.

(52) Campbell, E. M.; Hope, T. J. Live Cell Imaging of the HIV-1 Life Cycle. Trends Microbiol. 2008, 16, 580-587.

(53) Francis, A. C.; Melikyan, G. B. Live-Cell Imaging of Early Steps of Single HIV-1 Infection. Viruses 2018, 10, 275.

(54) McDonald, D.; Vodicka, M. A.; Lucero, G.; Svitkina, T. M.; Borisy, G. G.; Emerman, M.; Hope, T. J. Visualization of the Intracellular Behavior of HIV in Living Cells. J. Cell Biol. 2002, 159, $441-452$.

(55) Mamede, J. I.; Cianci, G. C.; Anderson, M. R.; Hope, T. J. Early Cytoplasmic Uncoating Is Associated with Infectivity of HIV-1. Proc. Natl. Acad. Sci. U. S. A. 2017, 114, E7169-E7178.

(56) Burdick, R. C.; Delviks-Frankenberry, K. A.; Chen, J.; Janaka, S. K.; Sastri, J.; Hu, W.-S.; Pathak, V. K. Dynamics and Regulation of Nuclear Import and Nuclear Movements of HIV-1 Complexes. PLoS Pathog. 2017, 13, No. e1006570.

(57) Francis, A. C.; Melikyan, G. B. Single HIV-1 Imaging Reveals Progression of Infection through CA-Dependent Steps of Docking at the Nuclear Pore, Uncoating, and Nuclear Transport. Cell Host Microbe 2018, 23, 536-548.

(58) Borrenberghs, D.; Dirix, L.; De Wit, F.; Rocha, S.; Blokken, J.; De Houwer, S.; Gijsbers, R.; Christ, F.; Hofkens, J.; Hendrix, J.; Debyser, Z. Dynamic Oligomerization of Integrase Orchestrates HIV Nuclear Entry. Sci. Rep. 2016, 6, 36485.

(59) Hendrix, J.; Gijsbers, R.; De Rijck, J.; Voet, A.; Hotta, J.; McNeely, M.; Hofkens, J.; Debyser, Z.; Engelborghs, Y. The Transcriptional Co-Activator LEDGF/P75 Displays a Dynamic Scan-and-Lock Mechanism for Chromatin Tethering. Nucleic Acids Res. 2011, 39, 1310-1325.

(60) Digman, M. A.; Gratton, E. Lessons in Fluctuation Correlation Spectroscopy. Annu. Rev. Phys. Chem. 2011, 62, 645-668.

(61) Ivanchenko, S.; Lamb, D. C. Fluorescence Correlation Spectroscopy: Principles and Developments. In Supramolecular Structure and Function 10; Brnjas-Kraljević, J., Pifat-Mrzljak, G., Eds.; Springer Netherlands: Dordrecht, 2011; pp 1-30.

(62) Digman, M. A.; Gratton, E. Analysis of Diffusion and Binding in Cells Using the RICS Approach. Microsc. Res. Tech. 2009, 72, 323332.

(63) Digman, M. A.; Wiseman, P. W.; Horwitz, A. R.; Gratton, E. Detecting Protein Complexes in Living Cells from Laser Scanning Confocal Image Sequences by the Cross Correlation Raster Image Spectroscopy Method. Biophys. J. 2009, 96, 707-716.

(64) Digman, M. A.; Sengupta, P.; Wiseman, P. W.; Brown, C. M.; Horwitz, A. R.; Gratton, E. Fluctuation Correlation Spectroscopy with a Laser-Scanning Microscope: Exploiting the Hidden Time Structure. Biophys. J. 2005, 88, L33-L36.

(65) Schrimpf, W.; Lemmens, V.; Smisdom, N.; Ameloot, M.; Lamb, D. C.; Hendrix, J. Crosstalk-Free Multicolor RICS Using Spectral Weighting. Methods 2018, 140-141, 97-111.

(66) Hendrix, J.; Dekens, T.; Schrimpf, W.; Lamb, D. C. ArbitraryRegion Raster Image Correlation Spectroscopy. Biophys. J. 2016, 111, 1785-1796.

(67) Digman, M. A.; Dalal, R.; Horwitz, A. F.; Gratton, E. Mapping the Number of Molecules and Brightness in the Laser Scanning Microscope. Biophys. J. 2008, 94, 2320-2332.

(68) Brown, C. M.; Dalal, R. B.; Hebert, B.; Digman, M. A.; Horwitz, A. R.; Gratton, E. Raster Image Correlation Spectroscopy (RICS) for Measuring Fast Protein Dynamics and Concentrations with a Commercial Laser Scanning Confocal Microscope. J. Microsc. 2008, 229, 78-91.

(69) Bag, N.; Wohland, T. Imaging Fluorescence Fluctuation Spectroscopy: New Tools for Quantitative Bioimaging. Annu. Rev. Phys. Chem. 2014, 65, 225-248. 
(70) Chen, Y.; Wu, B.; Musier-Forsyth, K.; Mansky, L. M.; Mueller, J. D. Fluorescence Fluctuation Spectroscopy on Viral-like Particles Reveals Variable Gag Stoichiometry. Biophys. J. 2009, 96, 1961-1969.

(71) Fogarty, K. H.; Chen, Y.; Grigsby, I. F.; Macdonald, P. J.; Smith, E. M.; Johnson, J. L.; Rawson, J. M.; Mansky, L. M.; Mueller, J. D. Characterization of Cytoplasmic Gag-Gag Interactions by DualColor Z-Scan Fluorescence Fluctuation Spectroscopy. Biophys. J. 2011, 100, 1587-1595.

(72) Hendrix, J.; Baumgärtel, V.; Schrimpf, W.; Ivanchenko, S.; Digman, M. A.; Gratton, E.; Kräusslich, H.-G.; Müller, B.; Lamb, D. C. Live-Cell Observation of Cytosolic HIV-1 Assembly Onset Reveals RNA-Interacting Gag Oligomers. J. Cell Biol. 2015, 210, 629-646.

(73) Derdowski, A.; Ding, L.; Spearman, P. A Novel Fluorescence Resonance Energy Transfer Assay Demonstrates that the Human Immunodeficiency Virus Type 1 Pr55Gag I Domain Mediates GagGag Interactions. J. Virol. 2004, 78, 1230-1242.

(74) Borrenberghs, D.; Thys, W.; Rocha, S.; Demeulemeester, J.; Weydert, C.; Dedecker, P.; Hofkens, J.; Debyser, Z.; Hendrix, J. HIV Virions as Nanoscopic Test Tubes for Probing Oligomerization of the Integrase Enzyme. ACS Nano 2014, 8, 3531-3545.

(75) Desimmie, B. A.; Weydert, C.; Schrijvers, R.; Vets, S.; Demeulemeester, J.; Proost, P.; Paron, I.; De Rijck, J.; Mast, J.; Bannert, N.; Gijsbers, R.; Christ, F.; Debyser, Z. HIV-1 IN/Pol Recruits LEDGF/P75 into Viral Particles. Retrovirology 2015, 12, 16.

(76) Borrenberghs, D.; Zurnic, I.; De Wit, F.; Acke, A.; Dirix, L.; Cereseto, A.; Debyser, Z.; Hendrix, J. Post-Mitotic BET-Induced Reshaping of Integrase Quaternary Structure Supports Wild-Type MLV Integration. Nucleic Acids Res. 2019, 47, 1195-1210.

(77) Del Portillo, A.; Tripodi, J.; Najfeld, V.; Wodarz, D.; Levy, D. N.; Chen, B. K. Multiploid Inheritance of HIV-1 during Cell-to-Cell Infection. J. Virol. 2011, 85, 7169-7176.

(78) Chen, B. K.; Saksela, K.; Andino, R.; Baltimore, D. Distinct Modes of Human Immunodeficiency Virus Type 1 Proviral Latency Revealed by Superinfection of Nonproductively Infected Cell Lines with Recombinant Luciferase-Encoding Viruses. J. Virol. 1994, 68, 654-660.

(79) Peng, K.; Muranyi, W.; Glass, B.; Laketa, V.; Yant, S. R.; Tsai, L.; Cihlar, T.; Müller, B.; Kräusslich, H.-G. Quantitative Microscopy of Functional HIV Post-Entry Complexes Reveals Association of Replication with the Viral Capsid. eLife 2014, 3, No. e04114.

(80) De Wit, F.; Pillalamarri, S. R.; Sebastian-Martin, A.; Venkatesham, A.; Van Aerschot, A.; Debyser, Z. Design of Reverse Transcriptase-Specific Nucleosides To Visualize Early Steps of HIV1 Replication by Click Labeling. J. Biol. Chem. 2019, 294, 1186311875 .

(81) Vansant, G.; Chen, H.-C.; Zorita, E.; Trejbalová, K.; Miklík, D.; Filion, G.; Debyser, Z. The Chromatin Landscape at the HIV-1 Provirus Integration Site Determines Viral Expression. Nucleic Acids Res. 2020, 48, 7801.

(82) Lucic, B.; Chen, H.-C.; Kuzman, M.; Zorita, E.; Wegner, J.; Minneker, V.; Wang, W.; Fronza, R.; Laufs, S.; Schmidt, M.; Stadhouders, R.; Roukos, V.; Vlahovicek, K.; Filion, G. J.; Lusic, M. Spatially Clustered Loci with Multiple Enhancers Are Frequent Targets of HIV-1 Integration. Nat. Commun. 2019, 10, 4059.

(83) Shehu-Xhilaga, M.; Crowe, S. M.; Mak, J. Maintenance of the Gag/Gag-Pol Ratio Is Important for Human Immunodeficiency Virus Type 1 RNA Dimerization and Viral Infectivity. J. Virol. 2001, 75, 1834-1841.

(84) Takagi, S.; Momose, F.; Morikawa, Y. FRET Analysis of HIV-1 Gag and GagPol Interactions. FEBS Open Bio 2017, 7, 1815-1825.

(85) Pelkmans, L.; Puntener, D.; Helenius, A. Local Actin Polymerization and Dynamin Recruitment in SV40-Induced Internalization of Caveolae. Science 2002, 296, 535-539.

(86) Nanbo, A.; Imai, M.; Watanabe, S.; Noda, T.; Takahashi, K.; Neumann, G.; Halfmann, P.; Kawaoka, Y. Ebolavirus Is Internalized into Host Cells via Macropinocytosis in a Viral GlycoproteinDependent Manner. PLoS Pathog. 2010, 6, No. e1001121.

(87) Qin, C.; Li, W.; Li, Q.; Yin, W.; Zhang, X.; Zhang, Z.; Zhang, X.-E.; Cui, Z. Real-Time Dissection of Dynamic Uncoating of
Individual Influenza Viruses. Proc. Natl. Acad. Sci. U. S. A. 2019, 116, $2577-2582$ 